\title{
An Estimate of the Integrated Risk of Flood and Earthquake in Santai County
}

\author{
Qiuyu Wang ${ }^{1,2,3}$, Chongfu Huang ${ }^{1,2,3, *}$ \\ ${ }^{1}$ Key Laboratory of Environmental Change and Natural Disaster, Ministry of Education, Beijing \\ Normal University, Beijing 100875, China \\ ${ }^{2}$ State Key Laboratory of Earth Surface Processes and Resource Ecology, Beijing Normal \\ University, Beijing 100875, China \\ ${ }^{3}$ Faculty of Geographical Science, Academy of Disaster Reduction and Emergency Management, \\ Beijing Normal University, Beijing 100875, China
}

\section{三台县洪水和地震综合风险的一个估计}

\author{
王秋余 ${ }^{1,2,3}$, 黄崇福 $1,2,3$, , \\ ${ }^{1}$ 北京师范大学, 环境演变与自然灾害教育部重点实验室, 北京100875, 中国 \\ ${ }^{2}$ 北京师范大学, 地表过程与资源生态国家重点实验室, 北京 100875 , 中国 \\ ${ }^{3}$ 北京师范大学, 地理科学学部, 减灾与应急管理研究院, 北京 100875 , 中国
}

\begin{abstract}
In essence, the key to solve the problem of multi-disaster probability risk assessment is to find out the joint probability distribution and its vulnerability function. Based on the principle of information diffusion, this paper proposes an integrated risk analysis model for earthquake and flood, the 2-dimension normal diffusion are employed to construct a discrete joint probability distribution and a disaster function, an integrated probability risk of multi-hazards is the expected value of disaster as a result. With building and ophiopogon as the subject matter of earthquake and flood disasters, applied in Santai county, Sichuan province, we calculate the Santai annual integrated risk is 584.5 million RMB,
\end{abstract}

*通讯作者: hchongfu@bnu.edu.cn

基金项目: 国家自然科学基金项目（编号: 41671502）, 国家重点研发计划（编号: 2017YFC1502902） accounting for $2.617 \%$ of the local GDP in 2016.

Keywords: information diffusion; earthquake; flood; integrated risk; Santai county

\section{摘要}

概率类多灾种综合风险评估问题本质上是 找出多灾种的联合概率分布和其脆弱性函 数。本文基于信息扩散原理提出地震和洪水 自然灾害综合风险分析模式, 通过对于表征 致灾因子的地震和洪水灾害二维信息扩散 概率密度函数与表征风险承受体的直接经 济损失脆弱性函数进行耦合, 最终得出以年 财产损失期望值为内涵的地震和洪水综合 风险值的评估结果。通过在四川省三台县进 行应用, 分别以建筑物和麦冬作为地震和洪 水灾害的标的, 计算出三台县的年化综合风 险值为 5.845 亿元，占当地 2016 年 GDP 的 2. $617 \%$ 。

关键词 : 信息扩散; 地震; 洪水; 综合风险; 三台县 
自然灾害是随机事件，多灾种综合风险 是多随机变量综合作用的产物。由于综合机 理十分复杂, 很难构造出有价值的理论分布 [1]。通常, 我们是在小样本的条件下进行综 合风险评估, 这就要求普适性的多灾种综合 风险评估方法, 必须具备优化处理不完备信 息的能力 ${ }^{[2]}$ 。

事实上, 综合风险评估就是找出一系列 的函数 [3], 例如概率密度函数和剂量-反应 曲线等, 并合成它们来显示风险 ${ }^{[4]}$ 。因此, 我们可以使用函数族和合成规则族符号来 建立这一基本模式。令 $R$ 是风险, $H$ 是描述 风险源的函数族, $D$ 是描述剂量-反应关系的 函数族, $\mathrm{o}$ 是合成规则族。任何风险, 包括 综合风险, 都可由式 $R=H \mathrm{o} D$ 所示的形式化 模型进行评估。该模型右边的三个部分分别 描述风险源、合成规则和剂量-反应关系。任 何现有的综合风险评估模型都只是它的一 个特例, 形式化模型的输入, 是与风险源因 素有关的参数; 输出是事件的可能性数值 ${ }^{[5]}$ 。 目前主要有三种被广泛使用的函数关系识 别工具 ${ }^{[6]}$ : 数学物理方程 ${ }^{[7]}$ 、统计回归 ${ }^{[8]}$ 和人 工神经元网络 ${ }^{[9]}$ 。虽然数学物理方程在结构 上最完美, 但使用条件最苛刻。对于统计回 归方法, 只有观察值总体类型已知, 并且样 本容量足够大, 其得出的函数关系才可靠。 而训练过的神经网络当作函数关系使用时, 它是一个黑盒子, 人们不可能知道它是如何 工作, 很难进行必要的修正, 故经常只能达 到局部最优的拟合, 而不能达到全局最优。 由于上述三种方法均不能很好地处理小样 本问题, 本文用信息扩散方法构造信息矩阵 进行函数关系的识别, 从而利用风险源和风 险承受体耦合出多灾种的综合风险。

\section{1 独立发生的洪水和地震财产损失综合 风险分析模型}

令 $W$ 是由致灾因子 $S$ 和 $Z$ 造成灾害 $D$ 的 $T$ 年内的观测样本。例如, $S$ 和 $Z$ 分别为 洪水和地震, $D$ 为财产损失。

当我们将两灾种 $S$ 和 $Z$ 的综合风险界定 为其在 $T$ 年内造成灾害 $D$ 的期望值时, 综合 风险评估的工作就是估计 $S$ 和 $Z$ 的联合概率 分布 $p(s, z)$ 和脆弱性函数 $d=f(s, z)[10]$, 从 而:

$$
r i s k=\int_{s_{0}}^{s_{\theta}} \int_{z_{0}}^{z_{\theta}} f(s, z) p(s, z) d x d z
$$

式中 $s_{0}$ 是能造成灾害的灾种 $\mathrm{S}$ 的最小值; $s_{\theta}$ 是在所研究地区灾种 $S$ 可能出现的最大值; $z_{0}$ 是能造成灾害的灾种 $Z$ 的最小值; $z_{\theta}$ 是所 研究地区灾种 $Z$ 可能出现的最大值。离散函 数的情况下:

$$
r i s k=\sum_{j=1}^{J} \sum_{k=1}^{K} d\left(u_{j}, v_{k}\right) P\left(u_{j}, v_{k}\right)
$$

\section{1 地震和洪水致灾因子联合概率分布的估计}

用前两个变量的观测样本估计联合概 率分布

$$
W_{1}=\left\{\left(s_{1}, z_{1}\right),\left(s_{2}, z_{2}\right), \ldots,\left(s_{n}, z_{n}\right)\right\}
$$

我们用 $\mathrm{S}$ 和 $\mathrm{Z}$ 分别表达两灾种的论域:

$$
\begin{gathered}
S=\left\{u_{j} \mid j=1,2, \ldots, J\right\} \\
Z=\left\{v_{k} \mid k=1,2, \ldots, K\right\}
\end{gathered}
$$

用 2 维正态扩散将 $W_{1}$ 在 $S \times Z$ 上进行扩 散处理

$$
\begin{aligned}
& \mu\left(\left(s_{i}, z_{i}\right),\left(u_{j}, v_{k}\right)\right)= \\
& \exp \left[-\frac{\left(s_{i}-u_{j}\right)^{2}}{2 h_{s}^{2}}\right] \exp \left[-\frac{\left(z_{i}-v_{k}\right)^{2}}{2 h_{z}^{2}}\right]
\end{aligned}
$$

式中 $h_{g}, \forall g \in\{s, z\}$, 是信息扩散系数, 根据 样本的大小取不同的值。其解析表达式如下:

$$
h_{g}= \begin{cases}0.8146\left(b_{g}-a_{g}\right), & n=5 \\ 0.5690\left(b_{g}-a_{g}\right), & n=6 \\ 0.4560\left(b_{g}-a_{g}\right), & n=7 \\ 0.3860\left(b_{g}-a_{g}\right), & n=8 \\ 0.3362\left(b_{g}-a_{g}\right), & n=9 \\ 0.2986\left(b_{g}-a_{g}\right), & n=10 \\ 2.6851\left(b_{g}-a_{g}\right) /(n-1), & n \geq 11\end{cases}
$$

式中

$b_{g}=\max _{1 \leq i \leq T}\left\{g_{i}\right\}, a_{g}=\min _{1 \leq j \leq T}\left\{g_{i}\right\}$.

再令

$$
Q_{j k}=\sum_{\mathrm{i}=1}^{\mathrm{T}} u\left(\left(s_{i}, z_{i}\right),\left(u_{j}, v_{k}\right)\right)
$$




$$
Q=\stackrel{u_{2}}{u_{1}} \begin{gathered}
v_{1} \\
u_{2} \\
u_{J}
\end{gathered}\left(\begin{array}{cccc}
Q_{11} & Q_{12} & \cdots & v_{k} \\
Q_{21} & Q_{22} & \cdots & Q_{1 K} \\
\vdots & \vdots & \ddots & \vdots \\
Q_{J 1} & Q_{J 1} & \cdots & Q_{J K}
\end{array}\right)
$$

归一化处理后估计得其联合概率分布:

$$
\mathrm{P}\left(u_{j}, v_{k}\right)=\frac{\sum_{i=1}^{T} u\left(\left(s_{i}, z_{i}\right),\left(u_{j}, v_{k}\right)\right)}{\sum_{j=1}^{J} \sum_{k=1}^{K} \Sigma_{i=1}^{T} u\left(\left(s_{i}, z_{i}\right),\left(u_{j}, v_{k}\right)\right)}
$$

\section{2 脆弱性函数的估计} 集合为

首先，假设在某一年的实际观测值样本

$$
\mathrm{W}=\left\{\left(s_{1}, z_{1}, d_{1}\right),\left(s_{2}, z_{2}, d_{2}\right), \ldots,\left(s_{n}, z_{n}, d_{n}\right)\right\}
$$

我们用 $S$ 和 $Z$ 分别表达两灾种的论域, 用 $D$ 表达财产损失的论域, 即

$$
\left\{\begin{array}{c}
S=\left\{u_{j} \mid j=1,2, \ldots, J\right\} \\
Z=\left\{v_{k} \mid k=1,2, \ldots, K\right\} \\
D=\left\{o_{l} \mid l=1,2, \ldots, L\right\}
\end{array}\right.
$$

用 3 维正态扩散将 $W$ 在 $S \times Z \times D$ 上依 照正态信息扩散公式 (3) 进行正态信息扩 散, 将其携带的信息扩散到论域中的所有点 上

$\mu\left(\left(s_{i}, z_{i}, d_{i}\right),\left(u_{j}, v_{k}, o_{t}\right)\right)=$

$\exp \left[-\frac{\left(s_{i}-u_{j}\right)^{2}}{2 h_{s}^{2}}\right] \exp \left[-\frac{\left(z_{i}-v_{k}\right)^{2}}{2 h_{z}^{2}}\right] \exp \left[-\frac{\left(d_{i}-o_{t}\right)^{2}}{2 h_{d}^{2}}\right]$

(8)

式中 $h_{g}, \forall g \in\{s, z, d\}$, 是信息扩散系数, 根 据样本的大小取不同的值, 仍由式 (4) 进 行计算。令

$$
\mathrm{Q}_{\mathrm{jkl}}=\sum_{\mathrm{i}=1}^{\mathrm{m}} \mu\left(\left(\mathrm{s}_{\mathrm{i}}, \mathrm{z}_{\mathrm{i}}, \mathrm{d}_{\mathrm{i}}\right),\left(\mathrm{u}_{\mathrm{j}}, \mathrm{v}_{\mathrm{k}}, \mathrm{o}_{\mathrm{l}}\right)\right)
$$

再令

$$
\begin{gathered}
H_{l}=\max _{\substack{1 \leq j \leq j \leq K \\
1 \leq k \leq K}}\left\{Q_{j k l}\right\} \\
r_{j k l}=\frac{Q_{j k l}}{H_{l}} \\
j=1,2, \ldots, J ; k=1,2, \ldots, K, \text { 则 } \\
\mathrm{R}=\left\{r_{j k l}\right\}_{J \times K \times L}
\end{gathered}
$$

是输入 $(s, z)$ 和输出 $d$ 之间的一个模糊关 系矩阵。
对于一个具有隶属函数 $\mu_{A}\left(u_{j}, v_{k}\right)$ 的模 糊输入 $\mathrm{A}$, 使用下面的近似推进公式, 可以 得到一个隶属函数为 $\mu_{B}\left(o_{l}\right), o_{l} \in D$ 的模糊输 出 $B$,

$$
\mu_{B}\left(O_{l}\right)=
$$

$\max _{1 \leq j \leq \jmath, 1 \leq k \leq K} \min \left\{\mu_{A}\left(u_{j}, u_{k}\right), r_{j k l}\right\}$

最后, 用重心法, 我们得到一个分明值: $d\left(u_{j}, v_{k}\right)=\left(\sum_{l=1}^{L} u_{B}\left(O_{l}\right) O_{l}\right) / \sum_{l=1}^{L} u_{B}\left(O_{l}\right)(14)$

它是用观测样本 $W$ 由三维正态信息扩 散构造的一个离散的脆弱性曲面。

\section{2 案例应用与讨论}

\section{1 三台县地震和洪水灾害现状}

四川省绵阳市三台县地处四川盆地中 东北部, 属川中丘陵地区, 地势北高南低。 人口 158 万, 是著名的农业大县。县境内大 小江河溪流 46 条, 均属于长江支流嘉陵江 水系, 其中涪江、凯江、梓江、郪江为四条 大江。随着人口的增加及河滩变迁, 该县的 洪水近年来有所增加, 2014 年 7 月的一次涪 江洪水, 两处桥梁冲毁, 使 1500 位村民身 陷 “孤岛”, 大片农田被毁。由于境内地质 构造简单, 曾被认为无地质断层, 但却在 5.12 汶川大地震后的 2009 年 6 月 30 日发生 了震中位于与三台县相临的德阳市绵竹市 5.6 级地震, 在 2013 年 2 月 19 日发生了震 中位于三台县、盐亭县交界处的 4.7 级地震, 于 2014 年 7 月 29 日发生了震中位于绵阳市 三台县的 4.6 级地震。仅以 2013 年 2 月 19 的地震为例, 三台县受灾人口 3920 人, 受 灾乡镇 14 个, 房屋受损 600 余间。 5.12 地 震中, 该县是地震烈度 VII 度区, 其北部城 镇新鲁镇属于重灾区, 境内土木结构房屋基 本尽数倒塌, 砖混结构房屋受损严重。

\section{2 三台县地震和洪水灾害风险评估}

\subsection{1 数据来源}

本文以四川三台县建筑物和主要经济 作物药材麦冬分别作为地震和洪水灾害的 风险承受体，采用了 2008-2016 年间中国地 震信息网、四川省三台县民政局提供的地震 震级和造成房屋经济损失数据, 三台县水务 局、麦冬办提供的年降雨量、造成麦冬经济 损失的数据, 如表 1 所示 
表 1 三台县洪水、地震经济损失表

\begin{tabular}{|c|c|c|c|c|c|}
\hline 年份 & $\begin{array}{c}\text { 年降雨量 } \\
\text { (毫米) }\end{array}$ & $\begin{array}{l}\text { 洪水造成麦冬 } \\
\text { 损失（万元） }\end{array}$ & $\begin{array}{l}\text { 地震 } \\
\text { 震级 }\end{array}$ & $\begin{array}{c}\text { 地震造成农村房 } \\
\text { 屋损失 } \\
\text { (万元 })\end{array}$ & $\begin{array}{c}\text { 总经济损失 } \\
\text { (万元) }\end{array}$ \\
\hline 2008 & 910.1 & 120 & 8 & 162200 & 162320 \\
\hline 2009 & 959.4 & 1235 & 5.6 & 400 & 1635 \\
\hline 2010 & 755.7 & 65 & 0 & 0 & 65 \\
\hline 2011 & 1152.9 & 1465 & 0 & 0 & 1465 \\
\hline 2012 & 929.1 & 735 & 0 & 0 & 735 \\
\hline 2013 & 988.4 & 1089 & 4.7 & 300 & 1389 \\
\hline 2014 & 630.7 & 90 & 4.6 & 800 & 890 \\
\hline 2015 & 1048.6 & 1214 & 0 & 0 & 1214 \\
\hline 2016 & 720.9 & 129.3 & 0 & 0 & 129.3 \\
\hline
\end{tabular}

\section{3 三台县洪水和地震综合风险的计算}

根据历史灾害数据, 获取三台县自 2008-2016 年的 9 个样本观测点, 属于小样 本, 即

$$
\begin{aligned}
\mathrm{W}= & \left\{\left(s_{1}, z_{1}, d_{1}\right),\left(s_{2}, z_{2}, d_{2}\right), \ldots,\left(s_{9}, z_{9}, d_{9}\right)\right\} \\
& =\{(910.1,8,162320),(959.4,5.6,1635),(7
\end{aligned}
$$

$55.7,0,65),(1152.9,0,1465),(929.1,0,735),(98$ $8.4,4.7,1389),(630.7,4.6,890),(1048.6,0,121$ 4), $(720.9,0,129.3)\}$

其中, 洪水、地震灾害致灾因子观测样本:

$$
\mathrm{W}=\left\{\left(s_{1}, z_{1}\right),\left(s_{2}, z_{2}\right), \ldots,\left(s_{9}, z_{9}\right)\right\}
$$

$$
=\{(910.1,8),(959.4,5.6),(755.7,0) \text {, }
$$

$(1152.9,0),(929.1,0),(988.4,4.7)$,

$(630.7,4.6),(1048.6,0),(720.9,0)\}$

年降雨量的最大值是 $1152.9 \mathrm{~mm}$, 最小 值是 $630.7 \mathrm{~mm}$, 故扩散系数

$v_{1}=0$
$u_{1}=500$
$u_{2}=630$
$u_{3}=760$
$u_{4}=890$
$u_{5}=1020$
$u_{6}=1150$
$u_{7}=1280$$\left[\begin{array}{lllllll}0.859 & 0.806 & 0.640 & 0.361 & 0.171 & 0.094 & 0.058 \\ 1.953 & 1.273 & 1.059 & 0.682 & 0.439 & 0.335 & 0.239 \\ 2.945 & 1.641 & 1.466 & 1.108 & 0.898 & 0.790 & 0.586 \\ 3.342 & 1.968 & 1.826 & 1.471 & 1.262 & 1.132 & 0.841 \\ 3.169 & 1.845 & 1.696 & 1.339 & 1.103 & 0.956 & 0.700 \\ 1.343 & 1.114 & 0.997 & 0.751 & 0.575 & 0.472 & 0.338 \\ & 0.401 & 0.349 & 0.250 & 0.177 & 0.136 & 0.095\end{array}\right]$

利用公式 (7) 可以得到离散的联合概率分布 $P$ :

$$
h_{S}=0.3362 \times(1152.9-630.7)=175.56
$$

对当地有影响的破坏性地震记录的最 大值是里氏 8 级, 最小值是里氏 4.5 级, 故 扩散系数

$$
h_{z}=0.3362 \times(8-4.6)=1.14
$$

因此, 洪水 $\mathrm{S}$ 和地震 $\mathrm{Z}$ 的离散论域取

$$
\begin{aligned}
\mathrm{S} & =\left\{u_{1}, u_{2}, \ldots, u_{7}\right\} \\
& =\{500,630,760,890,1020,1150,1280\} \\
\mathrm{Z} & =\left\{v_{1}, v_{2}, \ldots, v_{7}\right\} \\
& =\{0,5,5.75,6.5,7.25,8,8.75\}
\end{aligned}
$$

计算得到联合的信息矩阵 $Q$ : 


\begin{tabular}{|c|c|c|c|c|c|c|c|}
\hline & $v_{1}=0$ & $v_{2}=5$ & $v_{3}=5.75$ & $v_{4}=6.5$ & $v_{5}=7.25$ & $v_{6}=8$ & $v_{7}=8.75$ \\
\hline$u_{1}=500$ & -0.017 & 0.016 & 0.013 & 0.007 & 0.003 & 0.002 & 0.001 \\
\hline$u_{2}=630$ & 0.039 & 0.025 & 0.021 & 0.014 & 0.009 & 0.007 & 0.005 \\
\hline$u_{3}=760$ & 0.058 & 0.033 & 0.029 & 0.022 & 0.018 & 0.016 & 0.012 \\
\hline$u_{4}=890$ & 0.066 & 0.039 & 0.036 & 0.029 & 0.025 & 0.022 & 0.017 \\
\hline$u_{5}=1020$ & 0.063 & 0.037 & 0.034 & 0.027 & 0.022 & 0.019 & 0.014 \\
\hline$u_{6}=1150$ & 0.048 & 0.022 & 0.020 & 0.015 & 0.011 & 0.009 & 0.007 \\
\hline$u_{7}=1280$ & 0.027 & 0.008 & 0.007 & 0.005 & 0.003 & 0.003 & 0.002 \\
\hline
\end{tabular}

表 1 中, 地震和洪水的总体经济损失记 录最大最小值分别为 162320 万元和 65 万元, 由式 (4) 可计算出关于损失变量的扩散系 数为

$$
h_{d}=0.3362 \times(162320-65)=44464.131
$$

其离散论域可取

$D=\left\{d_{1}, d_{2}, \ldots, d_{7}\right\}$

$=\{0,27067,54234,81201,108368,135335,163402\}$

将三维信息矩阵转化为模糊关系矩阵, 并通过近似推理, 得到财产损失矩阵 LOSS。 这一矩阵亦可得由错误! 未找到引用源。

\begin{tabular}{c}
\multicolumn{2}{c}{$v_{1}=0$} & $v_{2}=5$ & $v_{3}=5.75$ & $v_{4}=6.5$ & $v_{5}=7.25$ & $v_{6}=8$ & $v_{7}=8.75$ \\
$u_{1}=500$ \\
$u_{2}=630$ \\
$u_{3}=760$ \\
$u_{4}=890$ \\
$u_{5}=1020$ \\
$u_{6}=1150$ \\
$u_{7}=1280$
\end{tabular}$\left[\begin{array}{ccccccc}46036.26 & 46679.42 & 47411.48 & 50899.44 & 63417.77 & 84290.83 & 96361.41 \\
46077.28 & 47034.37 & 48902.84 & 56493.75 & 74751.22 & 91804.75 & 98444.26 \\
46170.48 & 47583.05 & 50837.3 & 61733.51 & 80730.72 & 94112.93 & 98918.67 \\
46333.74 & 47859.5 & 51536.96 & 62929 & 81336.82 & 94066.24 & 98816.38 \\
46727.47 & 47414.27 & 49555.02 & 57214.03 & 73772.8 & 89886.01 & 97396.61\end{array}\right]$

脆弱性曲面示之。

从图 1 可以直观看出, 在不同震级的地 震和洪水的致灾强度下的当地建筑物和麦 冬的财产损失大小, 进而再将计算得到的离 散 $P$ 矩阵和 Loss 矩阵利用公式 (2) 进行耦 合, 可以计算出地震和洪水对民居和麦冬的 综合财产风险值为 5.845 亿元, 即四川省三 台县的地震和洪水灾害造成的建筑物和麦 冬的年化损失期望值是 5.845 亿元。另据《三 台县国民经济和社会发展统计公报》公示, 2016 年四川省三台县 GDP 达到 223. 36 亿元, 损失期望值占 GDP 的 $2.617 \%$ 。

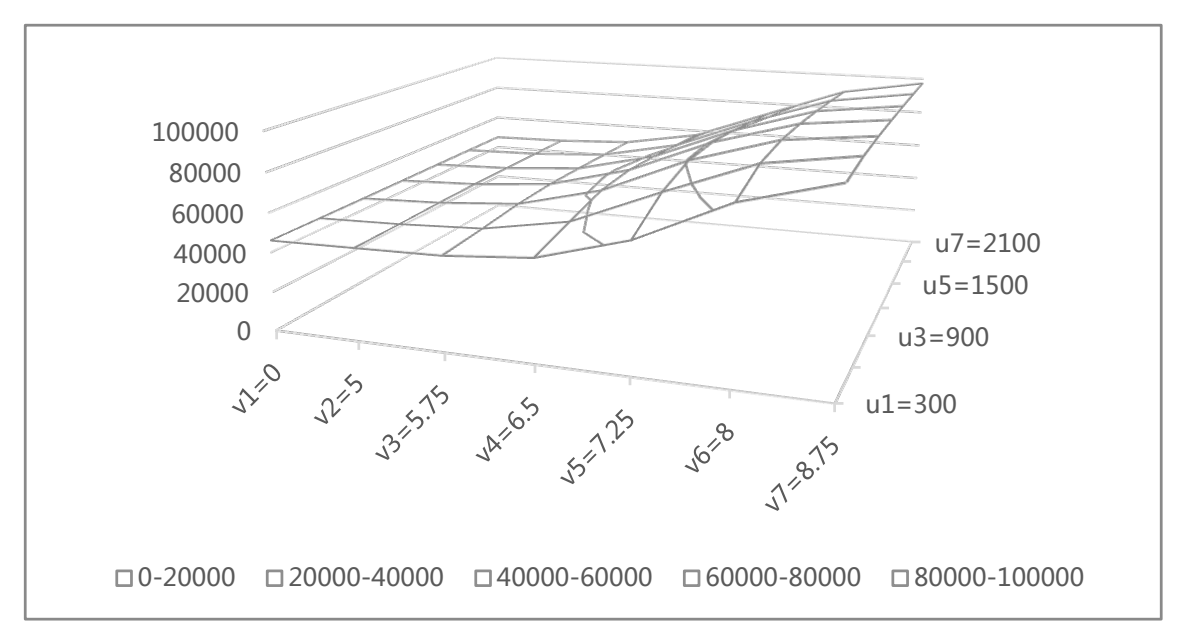

图 1. 四川省三台县关于地震和洪水的财产损失 (万元) 脆弱性曲面. $v$ 为震级, $u$ 为年降雨量 
严格来讲，根据表 1 所列历史灾害资料 计算出来的脆弱性, 并非风险承受体客观上 的脆弱性。依其计算出来的风险值, 只有参 考价值, 并无绝对的意义。

事实上, 对三台县造成最严重损失的 2008 的地震, 震中并非在该县。震中不同, 损失程度会有很大的差异。严格地计算关于 地震的脆弱性, 应该是用每次地震时当地的 地震波加速度场和损失分布场的数据来进 行。但这样的工作成本太高, 无法进行。

之所以计算结果有一定的参考价值, 是 因为, 如果的我们假定在未来的一些年中, 该区域的地震分布仍然没有大的变化, 县内 外的地震对该县的影响仍是如此, 则地震和 洪水灾害造成损失占 GDP 的 $2.617 \%$, 这一现 象不会有大的变化。

三台县地势北高南低, 大部分属于涪江 流域, 其主要经济作物受洪水灾害还是较为 严重, 加之部分毛坏房和土房子的存在导致 其受地震损失的风险也较大。政府管理部门 应做好对于地震和洪水灾害的避险宣传, 防 止麦冬减产，协同保险公司引导农户适当购 买农业保险; 对于毛坏房和土房子应尽快修 缮或重建, 保障人民群众的生命财产安全。

\section{3 结论}

本文基于信息扩散原理提出地震和洪 水自然灾害综合风险分析模式, 通过对于表 征致灾因子的地震和洪水灾害二维信息扩 散概率密度函数与表征风险承受体的直接 经济损失脆弱性函数进行耦合, 最终估计小 样本研究区一旦发生地震和洪水灾害, 其年 度财产损失的综合风险值。通过对四川省三 台县进行地震和洪水灾害综合风险的评估, 计算出其地震和洪水灾害对建筑物和麦冬 的年度财产损失期望值是 5.845 亿元, 占 2016 年 GDP 的 $2.617 \%$ 。这种模型对于风险 评估虽然只是一种比较粗糙的方式, 但在缺 乏大量可以统计分析的数据时, 依据反精确 原理它却是合适的。利用多维信息扩散方法 计算出的小样本综合风险, 对于以年为保险
期的财产保险, 具有一定的参考意义。

\section{参考文献}

[1] 庞西否.自然灾害动态风险分析基本模 式的探讨及其应用研究. 北京师范大学 博士学位论文, 2012.

[2] 薛晔,陈报章,黄崇福,等. 多灾种综合风 险评估软层次模型. 地理科学进展, 31 (3):353-360,2012.

[3] 黄崇福,综合风险评估的一个基本模式. 应用基础与工程科学学报,16(3):371-3 81,2008 .

[4] Wang W D, Qiao S, Zeng F L, Guo J, Chongfu Huang. An Approach to Ranking Integrated Models for Risk Assessment by Using the Internetof Intelligences, Journal of Risk Analysis and Crisis Response, 6(1): 2-9, 2016.

[5] 吴粀,黄崇福,翟国方,等. 基于综合风险 评估模式与信息扩散原理的台风灾害 风险评估方法探讨及应用，中国灾害防 御协会风险分析专业委员会第六届年 会论文集(呼和浩特, 2014 年 8 月 23-27 日).

[6] 黄崇福. 自然灾害风险分析与管理. 科 学出版社, 2012.

[7] 吴方同. 数学物理方程. 武汉大学出版 社, 2002 .

[8] 何晓群. 现代统计分析方法与应用. 中 国人民大学出版社, 2012.

[9] 张青贵. 人工神经网络导论. 水利水电 出版, 2004.

[10] Huang C F. An approach to assess an integrated risk caused by two hazards. Proceedings of the 6th International Conference on Risk Analysis and Crisis Response (June 5-9,2017, Ostrava-Prague, Czech), pp. 33-41. 